\title{
PROBLEMAS DE NOMENCLATURA EN LA CONSERVACIÓN Y RESTAURACIÓN DEL PATRIMONIO HISTÓRICO
}

\author{
Fernando Bolívar Galiano \\ Doctor en Bellas Artes. \\ Restaurador Biólogo. \\ Departamento de Pintura \\ Facultad de Bellas Artes \\ Universidad de Granada
}

El primer problema que se plantea en la denominación de la morfología de las alteraciones que determinan el estado de conservación de una obra artística, es la falta de acuerdo entre los diversos autores especializados. Las propuestas nomenclaturales, cada vez más abundantes, poseen notables diferencias conceptuales, que contribuyen a complicar la tarea de diagnosis del restaurador y/o investigador.

Este es el caso de términos de significado similar que son considerados como sinónimos por algunos trabajos más sinteticistas (Arnold et al., 1980; Ordaz y Esbert, 1988; CNR-ICR, 1990) o como conceptos totalmente diferenciados por autores más detallistas (Delgado Rodrigues, 1988; Alcalde y Martín, 1990). Nos referimos, por ejemplo, a los términos fisuración, fracturación, desintegración, disgregación... o incluso a las diferencias en la amplitud del concepto "pátina".

Mientras que algunos autores sólo incluyen en el término pátina las alteraciones superficiales de difícil explicación (Alcalde y Martín, 1990) y diferencian otros conceptos (limonitización, tinción, moteado, etc.), otros los definen como diversos tipos de pátinas: de rubefacción, de decoloración, de enmugrecimiento, de cromatización amarillenta y pátina de verdín (Esbert y Marcos, 1982). Por otro lado, en restauración de obras de arte en general, abarca gran cantidad de acepciones incluyendo los conceptos de incrustación, concreción, entre otros (Brachert, 1990).

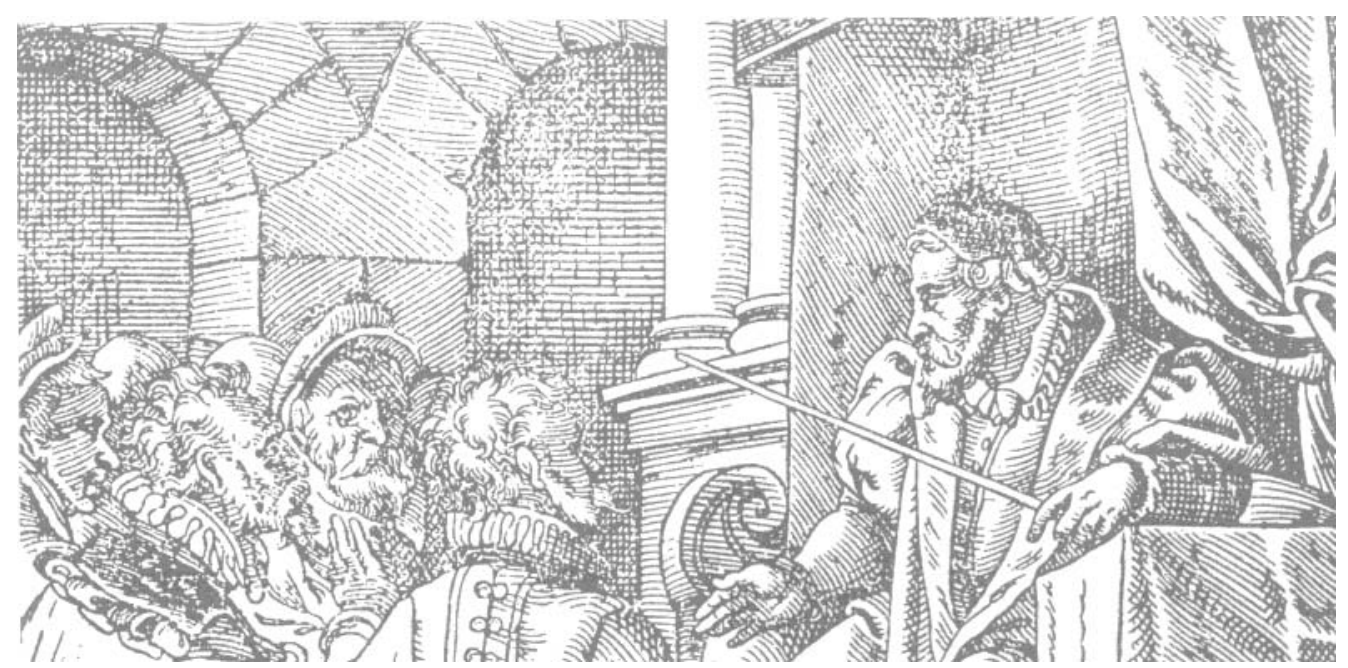

En algunos trabajos se ha utilizado el término "costra" en sentido amplio, que abarca no sólo a las modificaciones superficiales del propio material (CNR-ICR, 1990; Alcalde y Martín, 1990) sino también a los depósitos orgánicos o minerales que se encuentran más o menos adheridos a la superficie pétrea original (Bolívar Galiano, 1994). Este uso está fundamentado en la gran cantidad de formaciones vegetales criptogámicas y bacterianas, englobadas bajo el término "costras", que se estudian en numerosos trabajos científicos de microclimas epi y endolíticos muy similares a los existentes en nuestros monumentos lapídeos. Golubic (1982, 1985 y 1990), se ocupa de los estromatolitos recientes (costras mineralizadas) y los tapetes ("mats") que se producen en los ambientes marinos, incluyendo una iglesia de una localidad costera. Del mismo modo Friedmann (197| y 1972), estudia al microscopio electrónico de barrido las algas endolíticas formadoras de costras en ambientes desérticos, así como su ecología en desiertos de Norteamérica. Bell (1983, 1986 y 1993) estudia a fondo las comunidades criptoendólicas de otros desiertos áridos de América del Norte, y con otros autores realiza un nuevo estudio en el desierto de Colorado (Bell et al., I 986 y 1988). La formación de costras algales en desiertos cálidos es también estudiada por Campbell et al. (1989), Metting (199|), Sommerfeld y Bell (1991) y Johansen (1993). De la misma forma las costras fito-microbianas son estudiadas en los desiertos antárticos (Friedmann y Ocampo, 1976; Friedmann, 1982 y Friedmann et al., 1988 y 1993). 
Respeto a las pérdidas de materia, es práctica la diferenciación empleada por Alcalde y Martín, según el alcance en profundidad de esta alteración por pérdida de la cohesión Intergranular (desintegración o desagregación, menos profunda, disgregación, de mayor profundidad). Con una sola palabra se define tanto el tipo de alteración como la intensidad o gravedad de la misma.

En cambio, para las rupturas pueden ser más fácilmente aplicados los conceptos de Delgado Rodrigues (1988) sobre "fractura" y "fisura" que los diferencian por su extensión longitudinal (alcanzar o no los contornos del cuerpo en cuestión) y no por su distanciamiento o separación. Sí enriquece el glosario el término recogido por Alcalde y Martín: fragmentación. Los grupos metodológicos de biología, química y petrografía del "Consiglio Nazionale de Ricerca" (CNR) y el "Istituto Centrale del Restauro" (ICR), por el contrario, no hacen ninguna diferenciación entre los dos primeros términos y el tercero no lo recogen en su propuesta de 1990.

Con los términos concreción e incrustación las diferencias son muy importantes, y van desde quienes los emplean indistintamente hasta quienes consideran oportuno establecer claras diferencias y nuevos conceptos (como "acreción" en el caso de fuentes monumentales). Mientras que el término concreción está admitido de forma uniforme, incrustación sufre importantes cambios en su definición. CNR-ICR (1990) y Alcalde y Martín (1990) lo consideran como un depósito estratiforme, pero únicamente los dos últimos hacen hincapié en que pueda introducirse en la superficie de la piedra. La ambigüedad de estos dos términos hace necesaria, bajo mi punto de vista, la consideración de la introducción en el substrato original, como una condición necesaria para que exista una clara diferenciación entre los términos concreción e incrustación.

En relación a las alteraciones por delaminación, la propuesta de Delgado Rodrigues para el material granítico ofrece una gran variedad de matices: lasca, escama, esquirla, plaqueta y placa, que se diferencian por la extensión y espesor de la lámina. En el caso de los materiales calcáreos, podría ser suficiente la diferenciación de tres tipos de delaminaciones: película, escama y placa, que son los únicos que se definen en los trabajos del CNR-ICR y Alcalde y Martín (1990).
En definitiva, las diferencias existentes en las terminologías propuestas, obligan al usuario a tener que decidirse por alguna de ellas, que no coincidirán necesariamente con las que otros elijan, creando un clima de imprecisión y de libre interpretación. No obstante, también es cierto que cuando se trabaja en un tipo de obra con características muy peculiares, uno se ve obligado a considerar nuevos términos que no son normalmente empleados, si pretende definir y especificar con exactitud el tipo y la localización de las formas de alteración.

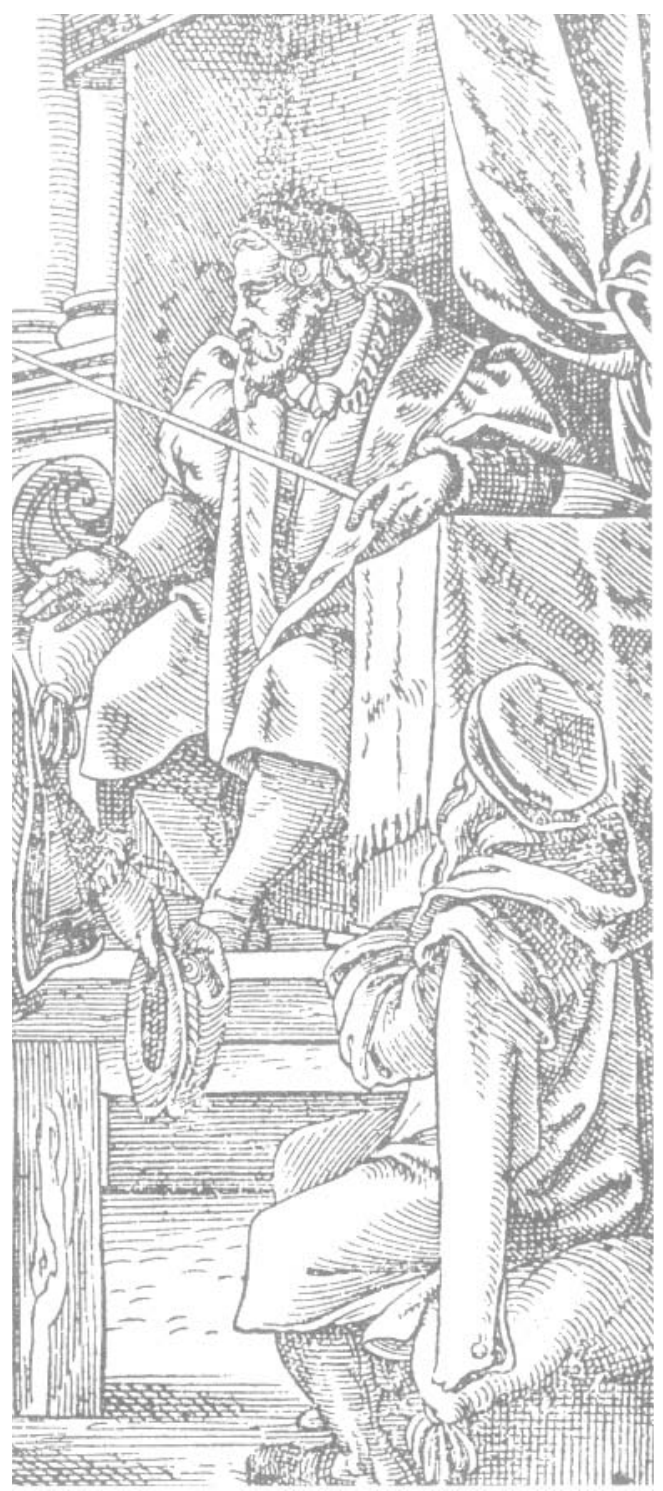

El primer

problema que se plantea en la denominación de la morfología de las alteraciones que determinan el estado de conservación de una obra artística, es la falta de acuerdo entre los diversos autores especializados 


\section{REFERENCIAS BIBLIOGRÁFICAS}

Alcalde, M. y A. Martín ( 1990): Indicadores visuales de alteración. En: Martín, A. Ensayos y experiencias de alteración en la conservación de obras de piedra de interés histórico-artístico. Ed. Centro de Estudios Ramón Areces. Madrid, 609 pags.

Arnold, A.; D. Jeanette y K. Zehnder ( 1980): Proposal for a terminology of weathering phenomena on building stones. Centre Documentation Iconos, pag: I-26.

Bell, R.A. (1986): "Endolithic algae of semi-desert sancistones: systematic, biogeographic and ecophysicologic investigations". PH. D. Thesis. Arizona state University, Tempe. 138 pag.

Bell. R.A. (1993): "Cryptoendolithic Algae of Hot semiarid Lands and Desert". Journal of Phycology, 29: 133-139.

Bell, R.A., P.V. Athey y M.R. Sommerfeld (1986): Cryptoendolithic algal comunities of the Colorado Plateau. Journal of Phycology, 22: 429-435.

Bell, R.A., P.V. Athey y Irl.R. Sommerfeld (1988): Distribution of endolithic algae on the Colorado Plateau of Northern Arizona. The Southwestern naturalist, 33 (3): 3। 5-322.

Bolívar Galiano, F. C. (1994): Diagnosis y tratamiento del deterioro por microalgas en los Palacios Nazaríes de la Alhambra. Tesis Doctoral. Universidad de Granada, 246 pag.

Brachert, Th. (1990): La patina nel restauro delle opere d'arte. Nardini editore. Edit. Ciudad. 237 pags.

Campbell, S.E.; J. Seeler y S. Golubic (1989): Desert crust formation and soil stabilization. Arid soil research and rehabilitation: 3: 217-228.

CNR-ICR (1990): Alterazioni macroscopiche dei materiali lapidei: lessico. Raccomandazioni Normal I/80. Gruppi B-C-P. Centro Stampa ICR, Roma. 36 pag.

Delgado Rodrigues, J. (199|): Proposal for a terminology of stone decay forms on monuments. G.P. News Letter. Group Petrography of the ICOMOS Stone Commitee. |/91. Lisboa; pag: 2-4.

Esbert, R.M.y R. Marcos (1982): La deterioración de las piedras de la Catedral de Oviedo. Formas y fenómenos de alteración. Materiales de Construcción. 186: 79-88.

Friedmann, E.I. (197I): Light and seanning electron microscopy of the endolithic desert algal habitat. Journal of Phycology, 10 (4): 4I I-428.
Friedmann, E.I. (1972): Ecology of lithophytic algal habitats in Middle Eastern and North American desert. In: Rodin, L.E. Ed. Ecophysiological Foundation of Ecosystems Productivity in Arid zones. Nauka USSR Acad. Sci. Leningrad, pag |82- | 85.

Friedmann, E.I. (1982): Endolithic microorganisms in the Antartic Cold Desert. Science. 2 1 5: I.045-1.053.

Friedmann, E.I. y R. Ocampo (1976): Endolithic Bluecreen Algae in the Dry Valleys: Primary producers in the Antartic Desert Ecosystem. Science. Ed. American Association for the Advancement of Science, vol 193: 1.247-1.249.

Friedmann, El., Irl. Hua y R. Ocampo (1988): Cryptoendolithic lichen Bell, R.A., P.V. Athey y M.R. Sommerfeld (1986): Cryptoendolithic algal comunities of the Colorado Plateau. Journal of Phycology, 22: 429-435.

Friedmann, L. Kappen, A.A. luleyer y J.A. Nienow (1993): Long-term Productivity in the Cryptoendolithic MicrobialCommunity of the Ross Desert, Antartida. Microbial Ecology, 25: 5I-69.

Golubic, S. (1982): Microbial ecology of algal mats and recent stromatolites in Shark Bay, Western Australia. National GeoRraphic Society Research Reports; | 4: 277-286.

Golubic, S. (1985): Microbial ni and modern stromatolites in Shark Bay, Western Australia. In: Caldwell D.E.; Brierley, J.A. y Brierley, C.L. (eds). Planetary ecology. Van Nostrand Reinhold. New York, pag: 3-I6.

Golubic, S. (1990): Modern Stromatolites: A Review. In: Robert Riding (Ed): Calcareous Algae and Stromatolites; 23: 54 I-56|

Johanaen, J.R. (1993): Cryptogamic crusts of semiarid and arid lands of North America. Journal of Phycology, 29: | 40- 147

Pletting, B. (1991): Biological surface features of semiarid lands and deserts. In: Skujins, I. (Ed). Semiarid lands and deserts: soil resource and reclamation. Marcel Dekker, pag: 257-293.

Ordaz, J. y n. Esbert (1988): Glosario de términos relacionados con el deterioro de las piedras de construcción. Materiales de construcción, vol. 38, n' 209: 39-45. 